\title{
Nanobiotechnology for the Detection and Control of Waterborne Parasites
}

\author{
Wafaa M. Hikal1,2*, Amra Bratovcic ${ }^{3}$, Rowida S. Baeshen1, Kirill G. Tkachenko4, \\ Hussein A. H. Said-Al Ahl5 \\ ${ }^{1}$ Department of Biology, Faculty of Science, University of Tabuk, Tabuk, KSA \\ ${ }^{2}$ Water Pollution Research Department, Environmental Research Division, National Research Centre, Giza, Egypt \\ ${ }^{3}$ Department of Physical Chemistry and Electrochemistry, Faculty of Technology, University of Tuzla, Tuzla, \\ Bosnia and Herzegovina \\ ${ }^{4}$ V. L. Komarov Botanical Institute of the Russian Academy of Sciences, Saint Petersburg, Russia \\ ${ }^{5}$ Medicinal and Aromatic Plants Research Department, National Research Centre, Giza, Egypt \\ Email: *wafaahikal@gmail.com
}

How to cite this paper: Hikal, W.M., Bratovcic, A., Baeshen, R.S., Tkachenko, K.G. and Said-Al Ahl, H.A.H. (2021) Nanobiotechnology for the Detection and Control of Waterborne Parasites. Open Journal of Ecology, 11, 203-223.

https://doi.org/10.4236/oje.2021.113016

Received: January 24, 2021

Accepted: February 23, 2021

Published: February 26, 2021

Copyright $\odot 2021$ by author(s) and Scientific Research Publishing Inc. This work is licensed under the Creative Commons Attribution International License (CC BY 4.0).

http://creativecommons.org/licenses/by/4.0/

\section{(c) (i) Open Access}

\begin{abstract}
Nowadays, the fast development of nanobiotechnology, has led to rapid diagnosis of important infectious diseases such as arboviruses-borne diseases, vector-borne infections and waterborne parasites diseases and others in order to reduce and avoid further dissemination of the infections within the general population. Furthermore, new nanomedicines based on the application of silver and gold nanoparticles which are less toxic, more effective, and that does not generate resistance could help to solve the problems of parasitic disease like leishmaniasis and chagas disease. It turns out that the combination of nanoparticles with antibiotics not only reduces the toxicity of both agents towards human cells but also enhances their ability to destroy bacteria by facilitating the binding of antibiotics to the microbes. Moreover, combining nanoparticles with antimicrobial peptides and essential oils with nanoparticles generates genuine synergy against microbial resistance.
\end{abstract}

\section{Keywords}

Parasites, Water, Nanobiotechnology, Control

\section{Introduction}

The applications of nanotechnology have made important developments in various fields. Here, we devote the study to the important developments brought about by nanotechnology and its application in two areas of great importance, 
which is known as nanomedicine, namely drug delivery-means the use of triggered response nanoparticulate systems and another emerging application of nanotechnology in medicine is its huge potential in diagnostics for the detection of biomarkers and pathogens. Figure 1 shows the graphical number of publications in the last twenty years.

\section{Parasites and Parasitic Diseases}

Parasites as hazardous microorganisms regarding human health might live within the host (endoparasites) or on the host surface (ectoparasites). The parasite organisms can cause many infectious diseases such as malaria, leishmaniasis, and trypanosomiasis, resulting in high morbidity and mortality in developing countries. Among them, malaria is caused by different parasite Plasmodium species [1], and is an acute public health problem that needs to be eliminated. Parasites include protozoa or helminths and arthropods including endoparasites/enteral, which inhabit body tissues inflicting serious health issues and ectoparasites are arthropods that either cause diseases, or act as vectors transmit alternative parasites [2].

Parasitic diseases are one of the world's most devastating and prevalent infections, causing millions of morbidities and mortalities annually. Most parasitic diseases and ways of their transmission are discovered and also known that environmental changes, human behavior, and population movement have an important impact on transmission, distribution, prevalence, and incidence of parasitic diseases [3].

Infectious diseases are mainly caused by pathogenic microorganisms including viruses, fungi, bacteria, and parasites that have a profound impact on humankind because of their unique characteristics such as the ability to rapidly

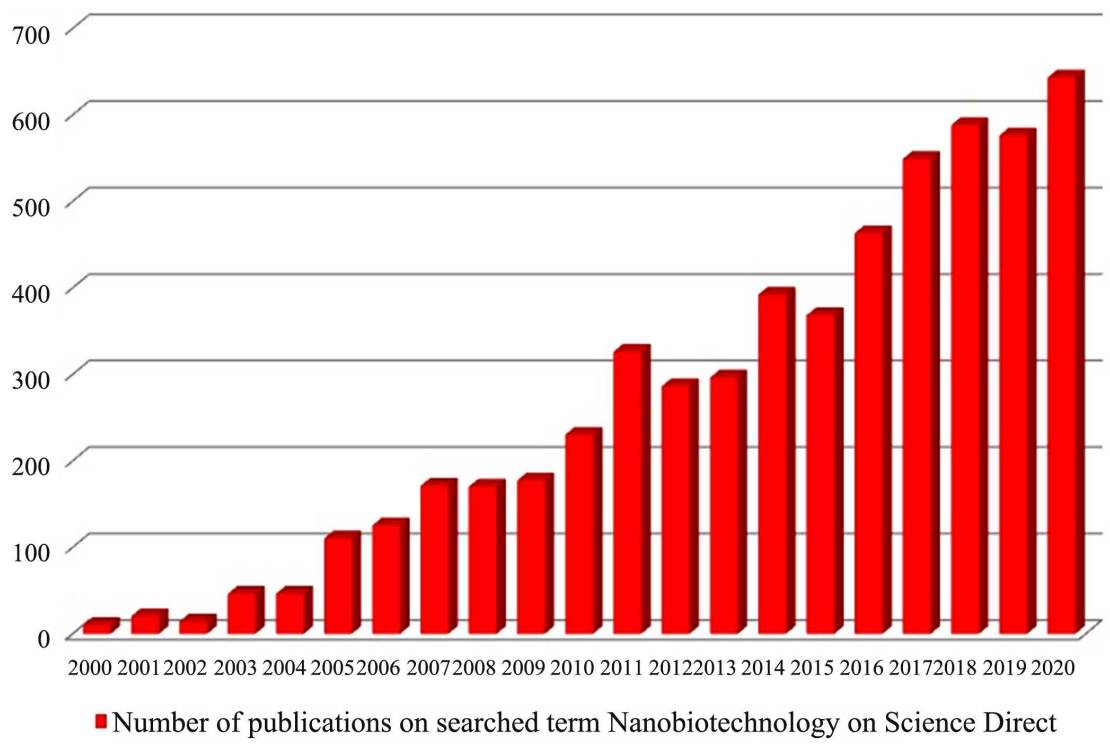

Figure 1. The graphical number of publications on Science Direct in the last twenty years for nanobiotechnology. 
multiply in number, unpredictability, and evolutionary advantage [1]. For instance, there were more than 229 million people infected with malaria around the world in 2019 and $~ 409,000$ patients died [4]. More importantly, the prevalent infectious diseases have caused a continuous increase in morbidity and mortality rates [5].

In the past, many of these infections have been linked predominantly to tropical or subtropical areas. Nowadays, however, climatic and vector ecology changes, a significant increase in international travel, armed conflicts, and migration of humans and animals have influenced the transmission of some parasitic diseases in developed countries. It has also been noted that many patients who have never traveled to endemic areas suffer from blood-borne infections caused by protozoa [6].

Thus, there is an urgent need to develop new diagnostics to detect infectious diseases to stop the spread, secure public health, and promote treatment. In addition, the ideal diagnostic tools for infectious diseases should be sensitive, rapid, specific, accurate, robust, low-cost, and user-friendly [7] [8]. Therefore, our research aims to shed light on previous studies in the field of using nanotechnology for the diagnosis and treatment of diseases.

\section{The Diagnosis Techniques}

For a long time, microscopy was the most popular approach to diagnose malaria, especially in developing countries [1]. Only a microscope and a drop of blood to check the malaria-induced parasites are required. However, this technology has many drawbacks for the detection of malarial disease. It is not easy to recognize different species of malarial parasites without skilled technicians. Low concentrations of parasites can also be extremely hard to diagnose. A few decades ago, PCR was demonstrated as the most efficient method for the detection of malaria with low levels of parasites [9]. However, it needs complicated operating skills and expensive equipment that is not usually available in poorly-resourced regions in developing countries. The diagnosis techniques like culture and microscopy, immunology, as well as polymerase chain reaction (PCR) strategies have significantly contributed to the diagnosis of infectious diseases and greatly promoted the prevention and treatment for various infectious diseases [1] [10] [11], but these techniques are slow, expensive, inaccurate, and require skilled technicians. Therefore, there is an urgent need to develop new technologies that help in early diagnosis and detection, with their high sensitivity [12]. The unique properties of nanomaterials or nanostructures confer the nanodiagnostic platforms on the ability of rapid and real-time detection by only using very small volumes of samples from patients.

\section{Nanotechnology and Nanoparticles}

Nanotechnological sciences (Nanoparticle-based Science) means technology usage of physical, chemical, or biological systems at scales starting from indi- 
vidual atoms or molecules to submicron dimensions, and has applications to our everyday activities encompasses the drug delivery and medical diagnostic research [13] [14] [15]. While, nanomedicine is the science and technology of designation, treating, and preventing illness and traumatic injury, relieving pain, and of conserving and up human health, exploitation molecular tools and molecular data of the human body [3].

Nowadays, nanotechnology-based anti-parasite pharmaceutical dosage forms development and application are being vastly developed worldwide. The field of nanotechnology involves an array of different areas of expertise with the application of innovative products in medicine, engineering, and to a less extent to veterinary medicine. New nanotechnological products like vaccines, target recombinant peptides, and other pharmaceutical alternatives enable a safer medium for animals and or humans. In this review, all aspects of nanoparticle applications in parasitology have been discussed [3].

Nanotechnology has advanced the development of many fields because of the unique properties of nanomaterials in biomedical applications including tissue engineering [16], drug delivery, bioimaging, and nanodiagnostics have been promoted by nanotechnology significantly [17] [18] [19] [20]. Nanodiagnostics applications have drawn more and more attention for infectious diseases because of their unique characteristics in early detection and high sensitivity.

Currently, antimicrobial resistance is a global public health threat that is leading us to new changes and the introduction of various fields in pursuit of novel and effective solutions [21] [22]. Nanomedicines are an important strategy for this, with several advantages in the development of antimicrobial agents [23].

Among the highest rates of morbidity and mortality worldwide are vector-borne infections such as malaria, yellow fever, dengue, Japanese encephalitis, filariasis, and others, which is considered dangerous to public health. Growing rates of resistance among arthropods to insecticidal substances and also serious environmental problems generated by overuse/misuse of insecticides make it difficult to effectively control the multiplication of arthropods and the infections caused by them. Therefore, a novel class of agents with high anti-arthropod activity and simultaneous minimal impact on the environment may help to improve the control of arthropods and arthropod-transmitted infections.

\section{Nanoparticles (NPs)}

Silver nanoparticles (Ag NPs) are one effective approach that has attracted the attention of researchers, that demonstrated remarkable antimicrobial properties and their activity against arthropods [23].

The use of noble metal nanoparticles, particularly gold and silver, in biomolecular applications has surged, ranging from innovative strategies for molecular diagnostics to radically new ways of treatment. Taking advantage of the particular optical-chemical characteristics of these metal NPs, every year new methods of molecular diagnostics of infectious diseases are reported providing higher 
analytical capability, sensitivity, and throughput at lower costs and with the possibility to be used where needed. Gold and silver NPs, or a combination of both, possess amazing optical/spectral properties [23]. Gold nanoparticles (Au NPs) are non-cytotoxic in nature with an additional advantage of a huge surface area, which makes their surfaces accessible for modification with targeting molecules, which make them advantageous over other nanoparticles for various biomedical applications. Targeted drug delivery is the most efficient therapy since it is possible to target only the affected cells or part. This minimizes the side effects of drugs. This is useful in treating cancer where the medicines can be delivered directly to the affected cells without damaging healthier cells in any way [24].

As multiresistant and pan-resistant infections continue to emerge, and because the development of novel antimicrobial drugs is a slow process, nanotechnology offers valuable alternatives for fighting resistant bugs, mainly by improving the therapeutic effect of current antimicrobials. Because of their physic and chemical properties, magnetite nanoparticles are the preferred vectors for many biomedical applications used in both diagnosis and therapy. Recent advances demonstrate that magnetic nanostructures may be used in anti-infectious therapy by enhancing the activity of natural and synthetic antimicrobial drugs [23].

Photodynamic therapy (PDT) is a growing field of interest as a potential alternative antimicrobial technique to antibiotics because of the widespread growth of drug resistance in pathogens. PDT involves the use of a dye called a photosensitizer (PS) that can be activated by light, causing the production of reactive oxygen species, which can destroy a wide array of different pathogenic microorganisms [23].

Metal nanoparticles (NP) are a promising antibiofilm agent [25]. They are already used as additives, mainly to medical devices and food packaging and preservation [26]. Among metal NPs, nanosilver is the most active and commonly used in medicine and hygiene. The roles of nanocarriers such as liposomes, polymeric nanoparticles (NPs), solid lipid NPs, and dendrimers in improving the delivery of antibiotics, reducing the minimum inhibitory concentration (MIC), and minimizing side effects are demonstrated. In addition, inorganic nanoparticles are effective both as nanobactericides ( $\mathrm{Ag}$ NPs, $\mathrm{ZnO} \mathrm{NPs}$, and $\mathrm{TiO}_{2} \mathrm{NPs}$ ) and as nanocarriers (Ag NPs, $\mathrm{Au}$ NPs, $\mathrm{ZnO} \mathrm{NPs}$, and $\mathrm{TiO}_{2} \mathrm{NPs}$ ) against sensitive and multi-drug-resistant bacterial strains.

The ongoing concern of antibiotic resistance in pathogenic microbes has raised the need for the development of new antimicrobial agents to control infectious diseases. The recent developments in nanotechnology have led to a range of nanomaterials that provide new opportunities to target pathogenic microbes. Silver nanoparticles (nano-Ag) represent antimicrobial agents with unique properties that improve their biocompatibility because of their high ratio of surface area to volume, no charge, and nontoxicity to humans. Some studies show that nano-Ag interact with membrane or cell wall, DNA, and proteins of microorganisms to produce the antimicrobial effect [23]. 
In this review, a brief account of the nanoparticle-based diagnosis and treatment for infectious diseases is provided, and our attention is focused on current and future research in this area to allow more rapid and accurate diagnoses, as well as to increase sensitivity in the identification of parasitic infections and new treatments.

\section{Nanoparticle-Based Diagnosis for Infectious Diseases}

There are many nanoparticles, particularly fluorescent nanoparticles, metallic nanoparticles, and magnetic nanoparticles, which have been successfully utilized for the diagnosis of infectious diseases. The most used metallic nanoparticles in the diagnosis applications are gold and silver nanoparticles. The gold nanoparticles are the first nanomaterials as nanodiagnostics for the detection of DNA in 1996 [27]. The changes in the color of gold nanoparticles in solution from red to blue have been demonstrated after DNA-guided aggregations, which make them ideal nanomaterials for nanodiagnostics because of their unique color changes and other chemical and physical properties. Many different molecules, such as antibodies, antigens, and enzymes, could be conjugated with gold nanoparticles as electrochemical labels, optical probes, and signal transfer amplifiers for the diagnosis of various diseases. Magnetic nanoparticles are nanocarriers that have been successfully applied in many biomedical applications, such as bioimaging, cancer therapy, and nanodiagnostics [28] [29] [30] [31] [32]. Iron oxide nanoparticles are used in order to detect many different infecting pathogens, such as viruses, bacteria, parasites, and for early diagnosis of malaria [33]. Figure 2 shows the graphical number of publications in the last fifteen years for the terms of nanotechnology on medicine, drug delivery, detection of biomarkers and parasitology.
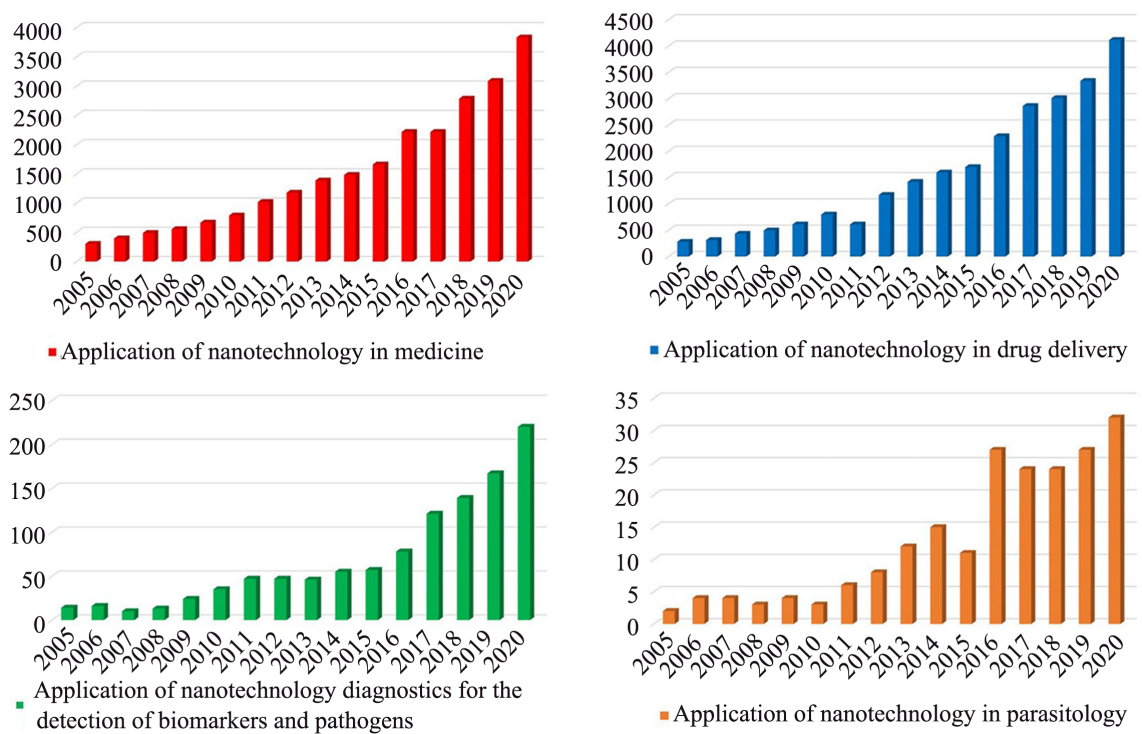

Figure 2. The graphical number of publications of nanotechnology on medicine, drug delivery, detection of biomarkers and parasitology in the last fifteen years. 
Malaria is an important parasitic infection caused by protozoan parasites the genus Plasmodium. This parasite causes major morbidity and mortality and is transmitted by female Anopheles mosquitoes. Figure 3 shows estimated malaria cases and deaths, 2010-2018 (world malaria report, 2019). Thus, methods for malaria diagnosis and treatment should be developed for saving human life. A colorimetric aptasensor for sensitive and specific detection of Plasmodium lactate dehydrogenase in malaria was designed by Jeon et al. [34]. Thus, the gold nanoparticles have obtained great attention as colorimetric reporters, due to their high extinction coefficients, ease of synthesis, and strongly distance-dependent optical characteristics. Colorimetric gold nanoparticles aptasensors have gained considerable attention for the detection of different targets because the molecular recognition events can be easily detected by the naked eyes without the need for any sophisticated equipment. Based on all that has been said, colorimetric gold nanoparticles aptasensors have great potential as commercial diagnostic tools. By using this system, the Plasmodium vivax and Plasmodium falciparum were determined [34].

Recently, Zheng and Huo [35] published a new rapid blood test for virus infection detection and diagnosis. They prepared a gold nanoparticle pseudo pathogen by coating citrate gold nanoparticles with a virus lysate. The mechanism of action is based on the reaction between the gold nanoparticle pseudovirus and the blood sample from a positive patient. The result of the reaction is the activation of immune molecules in the blood such as antibodies, complement proteins, and others leading to nanoparticle aggregate formation. The nanoparticle aggregate formation may be detected and measured using a dynamic light scattering technique. They applied this test for Zika virus infection detection. They tested blood plasma samples from 85 Zika positive patients, 40 Dengue positive patients, 10 Chikungunya positive patients, and 78 non-patient control samples collected from both endemic and non-endemic locations. The study shows that

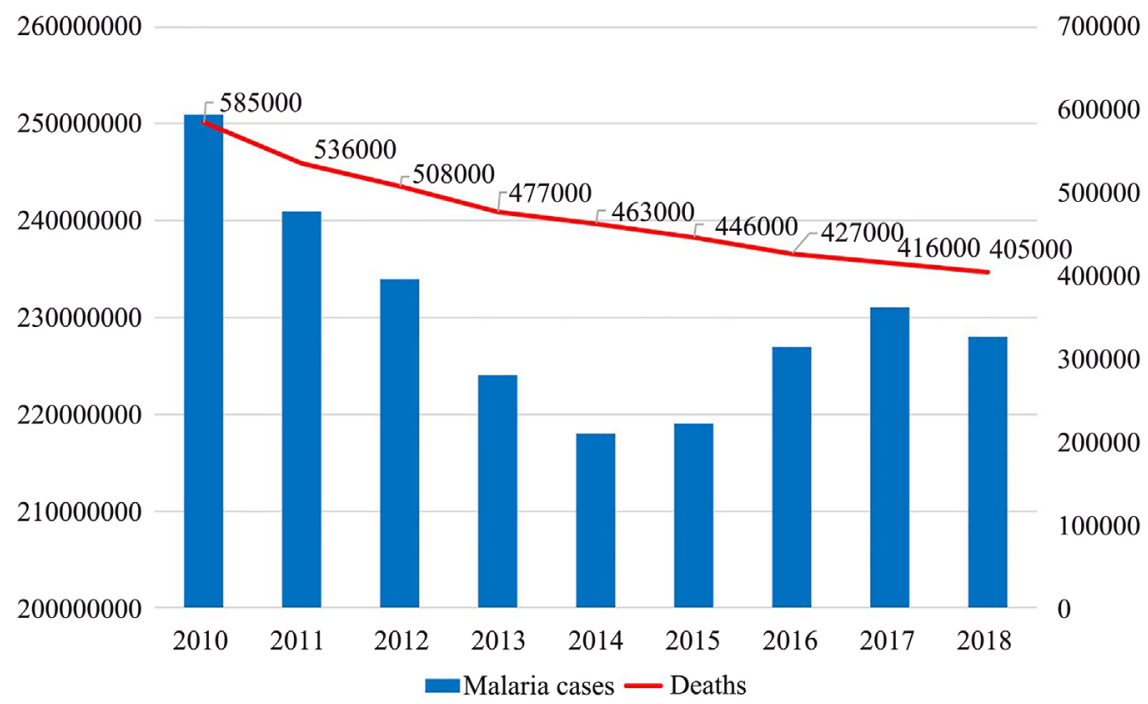

Figure 3. Estimated malaria cases and deaths, 2010-2018 (World Malaria Report, 2019). 
the new test has higher sensitivity compared to some existing commercial tests in the market while maintaining a similar specificity. For example, within 7 days from symptom onset, the new test detected $43 \%$ of the infected patients while a commercial anti-Zika IgM test detected only $26 \%$ of the infected patients. They highlight that the new test is extremely simple, easy to develop, with test results obtained within minutes. Moreover, this new test platform may be potentially adapted for the detection and diagnosis of a wide range of viral infectious diseases, for example, the currently ongoing COVID-19.

Krampa and co-workers [36] reported recent advances in the development of biosensors for Malaria diagnosis. They examined the various biosensor-based methods for malaria diagnostic biomarkers, Plasmodium falciparum histidine-rich protein 2 (PfHRP-2), parasite lactate dehydrogenase (pLDH), aldolase, glutamate dehydrogenase (GDH), and the biocrystal hemozoin.

\section{Nanoparticle-Based Treatment for Infectious Diseases}

The major therapeutic approach for infectious disease is the use of anti-microbial drugs. However, pathogens can become resistant, where anti-microbial drugs become therapeutically insufficient. In that case, administration dose and frequency are increased, resulting in increased side effects and toxicity. Besides, many pathogens are located intracellularly in an active or latent state, which prevents access to antimicrobial drugs [37]. The use of nano-delivery systems promises to overcome such limitations. Therefore, there is a growing interest to generate nanotechnology-based formulations against various pathogens, such as bacteria, viruses, fungi, or parasites. Application of nano-delivery for the treatment of infectious diseases includes both polymer-based and non-polymeric nanoparticles, as well as liposomes that improve the anti-microbial activity of drugs [37] [38].

Current research on these novel delivery systems has provided insight into possible indications, with evidence demonstrating their improved ability to specifically target pathogens, penetrate barriers within the host, and reduce toxicity with lower dose regimens. Nanoparticulate systems present a unique therapeutic alternative through the repositioning of existing medications and directed drug delivery [39].

Nanomedicine is the application of nanotechnology for the treatment, prevention, monitoring, and control of biological diseases. In applying nanomedicine in the treatment of diseases, the precise targets (cells and/or receptors) specific to the clinical disease are identified and the suitable nanoparticles for the delivery system to minimize the side effects and improve the efficacy of the original drug are selected. Several studies have explored the use of a nano-delivery system in improving the therapeutic efficacy of different drug molecules in the treatment of diseases. Mehrizi et al. [40] carried out the synthesis of a novel nanosized chitosan-betulinic acid delivery system, against resistant Leishmania, with the first clinical observation of this parasite in the kidney. It was discovered that chitosan nanoparticles synthesized using phase separation; and drug loading by phase separation, improved the therapeutic dose of betulinic acid to 20 
$\mathrm{mg} / \mathrm{kg}$. More, the successful improvement in the use of the nanosystem loaded betulinic acid in the treatment of Leishmania, displayed both in vitro and in vivo efficacy [40].

The effectiveness of ivermectin was investigated using nano structured lipid carriers in the treatment of hydatidosis, with some limitations and resistance associated with thedrug overcome by the carriers in in-vitro experimentation. It was observed that nanolipid carriers loaded ivermectin induced higher mRNAcaspase-3 expression which suggested a more potent apoptotic effect on the parasite [41]. In another related study, nucleoside-lipid-based nanocarriers were used to encapsulate methylene blue; a positively charged tricyclic phenothiazine molecule used in malaria treatment. This approach showed that the nanoparticles partially protected methylene blue from oxido-reduction reactions, thereby preventing early degradation during storage, and the carrier also prolonged the pharmacokinetics in plasma. This approach was an interesting technique in improving methylene blue stability and delivery in malaria treatment [42].

In recent decades, different types of nanostructures including nanotubes, nanodiamonds, thin films, nanorods, nanoparticles, nanofibers, and various clusters have been explored and applied in the construction of biosensors. Among nanomaterials mentioned above, gold nanoparticle as a new class of unique fluorescence quenchers is receiving significant attention in developing optical biosensors because of their unique physical, chemical, and biological properties. Yazdian-Robati et al. [43] used gold nanoparticles for the detection of antibiotics, toxins and infection diseases. The main factor that has contributed to the spread of Malaria disease is the increase in the number of drug-resistant parasites.

To overcome drug resistance, nanomaterials have been investigated for their capacity to effectively deliver antimalarial drugs at local doses sufficiently high to kill the parasites and avoid drug resistance evolution, while maintaining a low overall dose to prevent undesirable toxic side effects.

In recent years, several nanostructured systems such as liposomes, polymeric nanoparticles or dendrimers have been shown to be capable of improving the efficacy of antimalarial therapies [44]. A significant number of researchers have developed a drug delivery system using liposomes due to their dual hydrophilic/hydrophobic nature, they can encapsulate water-soluble drugs in the aqueous core and lipophilic compounds in the phospholipid bilayer. Dendrimers are among the most recent and attractive nanoparticulate systems, thanks to their multi-branched nanoarchitecture, which equipped them with multiple active sites for loading antibiotics and also interacting with bacteria [45].

Coma-Cros et al. [46] used the anionic copolymer Eudragit ${ }^{\oplus}$ S100 to assemble liposomes incorporating curcumin and containing either hyaluronan (Eudragit-hyaluronan liposomes) or the water-soluble dextrin Nutriose ${ }^{\varpi}$ FM06 (Eudragit-Nutriosomes). As a result, curcumin incorporated into Eudragit-Nutriosomes enhanced its antimalarial activity upon oral administration to Plasmodium yoelii-infected mice. Eudragit-Nutriosomes presented better performance than Eu- 
dragit-hyaluronan liposomes due to the greater stability of the former, as shown by the vesicle behavior in gastrointestinal fluids.

The drug delivery systems based on silica and liposomes were characterized by distinct features such as good biocompatibility, high percentage drug encapsulation, reduced drug toxicity, and targeted drug delivery [47]. Malaria, a mosquito-borne disease caused by Plasmodium species, claims more than 400,000 lives globally each year. The increasing drug resistance of the parasite renders the development of new anti-malaria drugs necessary. Varela-Aramburu et al. [48] tested glucose-based ultra-small gold nanoparticles that bind to cysteine-rich domains of Plasmodium falciparum surface proteins. Microscopy shows that ultra-small gold nanoparticles bind specifically to extracellular and all intra-erythrocytic stages of Plasmodium falciparum. Ultra-small gold nanoparticles may be used as drug delivery agents as illustrated for ciprofloxacin, a poorly soluble antibiotic with low antimalarial activity. Ciprofloxacin conjugated to ultra-small gold nanoparticles is more water-soluble than the free drug and is more potent. Glyco-gold nanoparticles that target cysteine-rich domains on parasites may be helpful for the prevention and treatment of malaria.

Schistosomiasis is a major parasitic disease that affects human health in tropical regions. Whilst Praziquantel is the main classic antischistosomal drug, new drugs are required due to the poor effect of the drug on the parasite juveniles and immature worms, and the emergence of drug resistant strains of Schistosoma. Nanotechnology is one of the most important recent and current methods used to treat human diseases including parasitic ones. Dkhil et al. [49] [50] showed that both gold and selenium nanoparticles reduced the levels of body weight changes, oxidative stress and histological impairment in the jejunal tissue significantly. Therefore, the results revealed the protective role of both selenium and gold nanoparticles against $S$. mansoni.

In vitro, Kar et al. [51] carried out a study that brings to the fore, the anthelmintic efficacy of gold nanoparticles. Gold nanoparticles were tested as vermifugal agents against a model cestode (Raillietina sp.) an intestinal parasite of domestic fowl. The study proved the effectiveness of gold nanoparticles in a plausible anthelmintic role and recommended further in-vivo studies are required on the anthelmintic efficacy of nanogold particles.

Undoubtedly, the gastrointestinal protozoan parasites particularly, Entamoeba histolytica and Cryptosporidium parvum are among the commonest causes of diarrheal illness particularly among children worldwide. Their prevalence in children with diarrhea is higher than in those without them. Nanoparticles have received more attention as antiparasitic agents. Saad et al. [52] tested silver and copper nanoparticles as antiparasitic activity against two of the most environmentally spread parasites in Egypt (Entamoeba histolytica and Cryptosporidium parvum). A significant reduction for cysts viability ( $\mathrm{p}>0.05)$ was observed for $\mathrm{CuO}$ nanoparticles against Entamoeba histolytica cysts and Ag nanoparticles against Cryptosporidium parvum oocysts. Moreover, $\mathrm{LC}_{50}-3 \mathrm{~h}$ of $\mathrm{CuO} \mathrm{NPs}$ for Entamoeba histolytica and Cryptosporidium parvum were 0.13 and $0.72 \mathrm{mg} / \mathrm{l}$, 
while Ag nanoparticles recorded 0.34 and $0.54 \mathrm{mg} / \mathrm{l}$, respectively. Accordingly, these nanoparticles could be suggested as a new nano form agent for the safe and effective treatment of Entamoeba histolytica and Cryptosporidium parvum parasites. In other words, this means the capability of $\mathrm{CuO}$ and $\mathrm{Ag}$ nanoparticles to inactivate Entamoeba histolytica and Cryptosporidium parvum cysts. The $\mathrm{CuO}$ nanoparticles and Ag nanoparticles may represent alternative drugs and water treatment for Entamoeba histolytica and Cryptosporidium parvum cysts. The treatment based on $\mathrm{CuO}$ and $\mathrm{Ag}$ nanoparticles has a very important role in overcoming amoebiasis and cryptosporidiosis.

Roy et al. [53] studied the antifilarial activity of biopolymer functionalized gold nanoparticles against the human filarial parasite (Wuchereria bancrofti) and Setaria cervi (bovine parasite). Gold nanoparticles exhibited superior antifilarial activity against both human and bovine filarial parasites, and are able to induce oxidative stress and apoptotic cell death in filarial parasites mediated through mitochondria. In addition, the synthesized nanomaterials appear to be nontoxic to the mammalian system.

Neglected tropical diseases comprise a group of seventeen infectious conditions endemic in many developing countries. Among these diseases are three of protozoan origin, namely leishmaniasis, chagas disease, and African trypanosomiasis, caused by the parasites Leishmania spp., Trypanosoma cruzi, and Trypanosoma brucei, respectively. These diseases have their own unique challenges which are associated with the development of effective prevention and treatment methods. Collectively, these parasitic diseases cause more deaths worldwide than all other neglected tropical diseases combined. Moreover, many current therapies for these diseases are limited in their efficacy, possessing harmful or potentially fatal side effects at therapeutic doses. It is therefore imperative that new treatment strategies for these parasitic diseases are developed. Nanoparticulate drug delivery systems have emerged as a promising area of research in the therapy and prevention of neglected tropical diseases. These delivery systems provide novel mechanisms for targeted drug delivery within the host, maximizing therapeutic effects while minimizing systemic side effects. Currently approved drugs may also be repackaged using these delivery systems, allowing for their potential use in neglected tropical diseases of protozoan origin [39] [54].

Adeyemi and co-workers [55] reported the anti-trypanosoma activity of inorganic gold and silver nanoparticles and their alloys. These findings add support to the in vitro antiparasitic action of gold and silver nanoparticles against Trypanosoma brucei brucei, Trypanosoma congolense and Trypanosoma evansi, and also indicated that nanoparticles may be effective against both extracellular and intracellular parasites, and encourage holding future investigations to determine the in vivo anti-Trypanosoma effectiveness of these gold and silver nanoparticles at higher doses are warranted. Nevertheless, the gold and silver nanoparticles exhibited promising and selective in vitro anti-Trypanosoma action. As in Figure 4, the parasites mentioned in the study. 


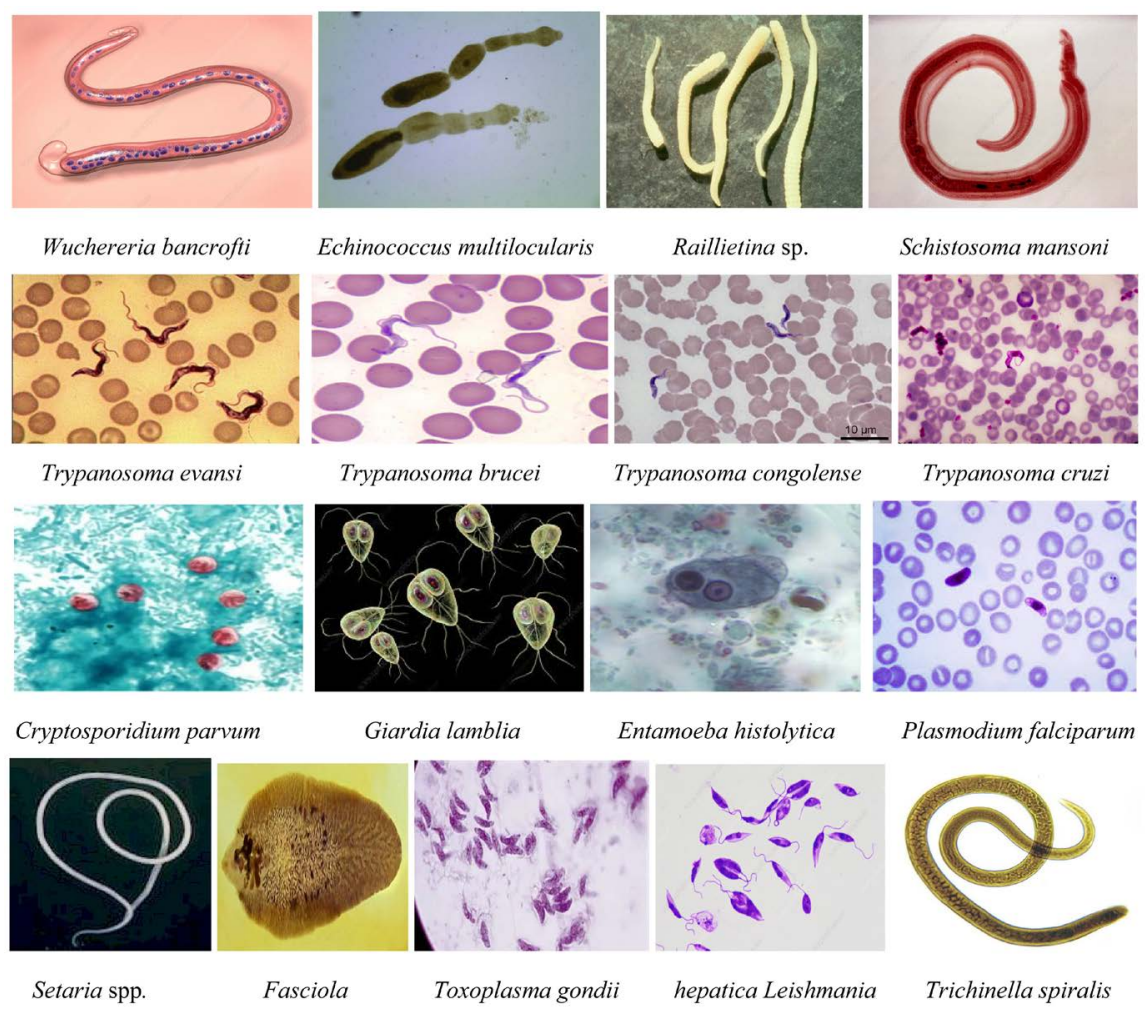

Figure 4. Different types of parasites.

Leishmaniasis is a widespread disease that causes 20,000 to 30,000 deaths annually, making it a major health problem in endemic areas. Because of low-performance medications, drug delivery poses a great challenge for better treatment of leishmaniasis. The present study's purpose was to review all relevant literature regarding the application of nanoparticles as a new method in leishmaniasis treatment and other parasites. In the present study, we tried to identify different research efforts in different conditions that examined the influence of various nanoparticles on different forms of leishmaniasis. In this way, we could compare their results and obtain a reliable conclusion from the most recent studies on this subject.

Silver nanoparticles (nano- $\mathrm{Ag}$ ) represent antimicrobial agents with unique properties that improve their biocompatibility because of their high ratio of surface area to volume, no charge, and nontoxicity to humans. Some studies show that nano-Ag interacts with membrane or cell wall, DNA, and proteins of microorganisms to produce the antimicrobial effect [56].

According to the results of Vazini [57] study, the $1000 \mathrm{ppm}$ of the gold nanoparticles can effectively use to eliminate the Leishmania major in both in vivo and in vitro. Thus, the metal nanoparticles such as gold, in suitable dosages, can be used for in vivo elimination of the promastigotes of Leishmania major. In addition, these results are considered as asuitable approach to find the proper alternative to the drugs used as a therapeutic agent for the cutaneous leishmaniasis caused by Leishmania major since it can be a method without the issues related 
to the chemical drugs such as glucantime.

Leishmaniasis and chagas disease, The most important protozoan infections caused by intracellular parasites. Treatments of chagas disease and cutaneous leishmaniasis have limitations including toxicity, variable efficacy, requirements for parenteral administration, and lengthy treatment regimens. Despite progress in visceral leishmaniasis treatments, the high cost is its main limitation. No preventive and therapeutic vaccine exists for these diseases. An attractive alternative to the urgent need of developing new medication alternatives to current treatments that are less toxic, more effective, and that does not generate resistance is offered by the intervention of nanotechnology in the pharmaceutical and immunological field. Nanomedicines could help solve the problems posed by the treatment and prophylaxis of parasitic diseases like leishmaniasis and chagas disease [54] [58].

Another study on Leishmania donovani, Baranwal et al. [59] reported that nanotherapeutic agents play a crucial role in clinical medicine. The antileishmanial potential of $\mathrm{Ag}$ nanoparticles was examined on the clinical isolates of Leishmania donovani promastigote cells in an in vitro experimental setting. Dose-dependent killing activity of the Ag nanoparticles was observed with an $\mathrm{IC}_{50}$ value of $51.88 \pm 3.51 \mu \mathrm{g} / \mathrm{ml}$. These results were also compared using the commercially available drug, miltefosine. Furthermore, the clinical applicability of Ag nanoparticles, as an antileishmanial agent was proven by testing them against normal mammalian monocyte cell line (U937), and no significant toxicity of Ag nanoparticles on the normal mammalian cells was observed [59].

Moreover, Amphotericin B (AmB) as a liposomal formulation of AmBisome is the first line of treatment for the disease, visceral leishmaniasis, caused by the parasite Leishmania donovani. However, nephrotoxicity is very common due to poor water solubility and aggregation of Amphotericin. So, Kumar et al. [60] developed a water-soluble covalent conjugate of gold nanoparticles with a polyene antibiotic Amphotericin B for improved antileishmanial efficacy and reduced cytotoxicity. Macrophages treated with gold nanoparticles with Amphotericin $B$ showed increased immunostimulatory $T_{h} 1$ (IL-12 and interferon- $\gamma$ ) response compared with standard Amphotericin B. In parallel, Amphotericin B uptake was $\sim 5.5$ and $\sim 3.7$-fold higher for gold nanoparticle-Amphotericin treated macrophages within 1 and $2 \mathrm{~h}$ of treatment, respectively. The ergosterol content in gold nanoparticles with Amphotericin treated parasites was almost two-fold reduced compared with Amphotericin B treated parasites. Moreover, gold nanoparticle with Amphotericin B was significantly less cytotoxic and hemolytic than Amphotericin B. According to these results, gold nanoparticle-based delivery of Amphotericin B can be a better, cheaper, and safer alternative than available Amphotericin B formulations [60].

Toxoplasmosis, a common food and pet-borne illness linked to hallucinations, personality alteration, and it's often carried by house pets. The treatment, developed at the University of Technology Sydney, Australia, uses gold nanoparticles that attach to toxoplasmid-hunting antibodies. The gold carrying-antibodies 
then spread through the circulatory system, affixing themselves to parasites in the blood. Once the gold particles are well distributed and widely attached to the parasite, the laser heats up the gold, incinerating the parasites [61]. Abdulsattar and Drywall [62] found that gold and nickel nanoparticles decrease the level of the enzyme by $41.3 \%$ and inhibition of nickel NPs was greater than $43.8 \%$. The results indicated that both IgA and IgG were shown to be inhibitory in the presence of nickel nanoparticles while the results showed activation of the immunoglobulin M (IgM) level with nickel nanoparticles.

In the last twenty-five years, a large number of studies have been carried out and many research groups have proven the efficiency of nanomaterials as anti-parasites [52] [63]-[80]. Studies were performed in vitro and/or in vivo. Said and co-workers [63] using Silver, chitosan, and curcumin nanoparticles on Giardia lamblia in vivo found that the highest fighter effect was achieved by combining the three nanoforms. The parasite was found to be eradicated from stool and the intestine. Another research carried out using Gold NPs Giardia lamblia in vitro, Bavand et al. [64] found that Gold NPs at a concentration of 0.3 $\mathrm{mg} \cdot \mathrm{ml}^{-1}$ can be used as an effective combination for killing Giardia cysts.

Saad et al. [52] using $\mathrm{CuO}$ (copper oxide) and $\mathrm{Ag}$ (silver) on E. histolytica, $C$. parvum in vitro and found that the treatment based on $\mathrm{CuO}$ NPs and Ag NPs showed a very important role in overcoming amoebiasis and cryptosporidiosis. Sedighi et al. [65] using Nano-Nitazoxanide (NTZ) on Cryptosporidium parvum in vivo and found that nano-nitazoxanide was effective on parasites on day 6.

Allahverdiyev et al. [66] using silver (Ag-NPs) on Leishmania tropica in vitro and found that Ag-NPs demonstrated significant antileishmanial effects by inhibiting the proliferation and metabolic activity of promastigotes. Allahverdiyev et al. [67] using $\mathrm{TiO}_{2}$ and $\mathrm{Ag}_{2} \mathrm{O}$ Leishmania in vitro and found that $\mathrm{TiO}_{2}$ and $\mathrm{Ag}_{2} \mathrm{O}$ NPs showed significant antibacterial activity. Sazgarnia et al. [68] using Gold NPs (GNPs) on Leishmania major in vitro and found that the presence of GNPs during MW irradiation was more lethal for promastigotes and amastigotes in comparison to MW alone. Venier-Julienne et al. [69] using Amphotericin B incorporated into poly (D,L-lactide-co-glycolide) on Leishmania in vitro and found that anti-leishmanial activity was observed with drug-free NPs. Salah-Tazdaï et al. [70] using Chitosan Leishmania infantum in vitro and found that Chitosan had not antileishmanial activity against Leishmania infantum LIPA 155/10. Karimi et al. [71] using silver NPs on Leishmania major in vitro and found that AgNPs alone did not kill Leishmania major promastigotes completely, while the combined use of both direct current electricity and AgNPs has a significant synergistic effect on promastigote mortality. Khosravi et al. [72] using silver NPs on Leishmania tropica in vitro found that the $\mathrm{IC}_{50}$ for nanosilver solutions was high significantly $\left(14.9 \mu \mathrm{g} \cdot \mathrm{ml}^{-1}\right)$. Jameii et al. [73] using selenium and silver on Leishmania major in vivo and found that unlike selenium NPs, Ag NPs showed an anti-Leishmanial effect in vivo.

Gaafar et al. [74] used chitosan and silver Toxoplasma gondii in vivo. The results showed that used AgNPs singly or combined with chitosan have promising 
anti-toxoplasma potentials.

Mohapatra et al. [75] using copper (II) nanohybrid solids, Lcu $\left(\mathrm{CH}_{3} \mathrm{COO}\right)_{2}$ and $\mathrm{LCuCl}_{2}$ on Plasmodium falciparum in vitro and found that the two compounds showed significant antimalarial activities against the parasites. Tripathy et al. [76] using chitosan-tripolyphosphate conjugated chloroquine on Plasmodium berghei in vivo and found that the maximum effect of nanoconjugated chloroquine (Nch) was found at $250 \mathrm{mg} \mathrm{kg}^{-1} \mathrm{bw}$ concentration during 15 days of treatment. Ponarulselvam et al. [77] on Plasmodium using silver NPs on Plasmodium falciparum in vitro and found that The Ag NPs showed antiplasmodial activity against $P$. falciparum. Nayak et al. [78] used curcuminoids-loaded lipid on Plasmodium berghei in vivo and found that the in vivo pharmacodynamic activity revealed a 2 -fold increase in antimalarial activity of curcuminoids entrapped in lipid NPs.

Abulaihaiti et al. [79] using Albendazole-chitosan microspheres on Echinococcus multilocularis in vivo and found that metacestode grown was highly suppressed during treatment with ABZ-CS-MPs. Brodaczewska et al. [80] using chitosan on Trichinella spiralis in vitro and found that although chitosan stimulated the lymphocyte response, the effect of treatment was not protective. Gherbawy et al. [81] using silver NPs on Fasciola in vitro and in vivo and found the percentage of non-hatching eggs treated with the triclabendazole drug was $69.67 \%$, while this percentage increased to $89.67 \%$ in combination with drug and AgNPs.

\section{Conclusion}

Thanks to development research in the field of bionanotechnology, the incorporation of nanoparticles with chemical drugs, peptides, and essential oils, the quality, efficiency, and sustainability of drugs have been significantly improved and their costs have been reduced. Moreover, considering the use of nanoparticles in the destruction of parasites, their inhibitory effect (making drugs more effective and less harmful), and their utility in making effective vaccines to prevent and fight against parasites, further research on this issue is highly recommended.

\section{Conflicts of Interest}

The authors declare no conflicts of interest regarding the publication of this paper.

\section{References}

[1] Wang, Y., Yu, L., Kong, X. and Sun, L. (2017) Application of Nanodiagnostics in Point-of-Care Tests for Infectious Diseases. International Journal of Nanomedicine, 12, 4789-4803. https://doi.org/10.2147/IJN.S137338

[2] Hikal, W.M. (2020) Parasitic Contamination of Drinking Water and Egyptian Standards for Parasites in Drinking Water. Open Journal of Ecology, 10, 1-21. https://doi.org/10.4236/oje.2020.101001 
[3] Farhoudi, R. (2017) An Overview on Recent New Nano-Anti-Parasitological Findings and Application. Advances in Nano Research, 5, 49-59. https://doi.org/10.12989/anr.2017.5.1.049

[4] World Health Organization (2020) World Malaria Report 2020. https://www.who.int/teams/global-malaria-programme/reports/world-malaria-repo rt-2020

[5] Kotloff, K.L., Nataro, J.P., Blackwelder, W.C., Nasrin, D., Farag, T.H., Panchalingam, S., et al. (2013) Burden and Aetiology of Diarrhoeal Disease in Infants and Young Children in Developing Countries (the Global Enteric Multicenter Study, GEMS): A Prospective, Case-Control Study. Lancet, 382, 209-222. https://doi.org/10.1016/S0140-6736(13)60844-2

[6] Momcilovic, S., Cantacessi, C., Arsić-Arsenijević, V., Otranto, D.S. and Tasić-Otašević, S. (2019) Rapid Diagnosis of Parasitic Diseases: Current Scenario and Future Needs. Clinical Microbiology and Infection, 25, 290-309.

https://doi.org/10.1016/j.cmi.2018.04.028

[7] Fauci, A.S. and Morens, D.M. (2012) The Perpetual Challenge of Infectious Diseases. New England Journal of Medicine, 366, 454-461. https://doi.org/10.1056/NEJMra1108296

[8] Hauck, T.S., Giri, S., Gao, Y. and Chan, W.C.W. (2010) Nanotechnology Diagnostics for Infectious Diseases Prevalent in Developing Countries. Advanced Drug Delivery Reviews, 62, 438-448. https://doi.org/10.1016/j.addr.2009.11.015

[9] Tham, J.M., Lee, S. H., Tan, T.M.C., Ting, R.C.Y. and Kara, U.A.K. (1999) Detection and Species Determination of Malaria Parasites by PCR: Comparison with Microscopy and with Para Sight-F and ICT Malaria Pf Tests in a Clinical Environment. Journal of Clinical Microbiology, 37, 1269-1273. https://doi.org/10.1128/JCM.37.5.1269-1273.1999

[10] Wilson, M.R., Naccache, S.N., Samayoa, E., Biagtan, M., Bashir, H. and Yu, G. et al. (2014) Actionable Diagnosis of Neuroleptospirosis by Next-Generation Sequencing. New England Journal of Medicine, 370, 2408-2417. https://doi.org/10.1056/NEJMoa1401268

[11] Golding, C.G., Lamboo, L.L., Beniac, D.R. and Booth, T.F. (2016) The Scanning Electron Microscope in Microbiology and Diagnosis of Infectious Disease. Scientific Reports , 6, Article No. 26516. https://doi.org/10.1038/srep26516

[12] Laksanasopin, T., Guo, T.W., Nayak, S., Sridhara, A.A., Xie, S., Olowookere, O.O., et al. (2015) A Smartphone Dongle for Diagnosis of Infectious Diseases at the Point of Care. Science Translational Medicine, 7, $273 \mathrm{re} 1$. https://doi.org/10.1126/scitranslmed.aaa0056

[13] Bhushan, B., Tang, W. and Ge, S. (2010) Nanomechanical Characterization of Skin and Skin Cream. Journal of Microscopy, 240, 135-144. https://doi.org/10.1111/j.1365-2818.2010.03407.x

[14] Arangoa, M.A., Campanero, M.A., Renedo, M.J., Ponchel, G. and Irache, J.M. (2001) Gliadin Nanoparticles as Carriers for the Oral Administration of Lipophilic Drugs. Relationship between Bioadhesion and Pharmacokinetics. Pharmaceutical Research, 18, 1521-1527. https://doi.org/10.1023/A:1013018111829

[15] Oowaki, H., Matsuda, S., Sakai, N., Ohta, T., Iwata, H., Sadato, A., Taki, W., Hashimoto, N. and Ikada, Y. (2000) Non-Adhesive Cyanoacrylate as an Embolic Material for Endovascular Neurosurgery. Biomaterials, 21, 1039-1046. https://doi.org/10.1016/S0142-9612(99)00278-1

[16] Bratovcic, A. (2020) Nanocomposite Hydrogels Reinforced by Carbon Nanotubes. 
International Journal of Engineering Research and Applications, 10, 30-41.

[17] Chai, F., Sun, L., Ding, Y., Liu, X., Zhang, Y., Webster, T.J. and Zheng, C. (2016) A Solid Self-Nanoemulsifying System of the BCS Class IIb Drug Dabigatran Etexilate to Improve Oral Bioavailability. Nanomedicine, 11, 1801-1816.

https://doi.org/10.2217/nnm-2016-0138

[18] Sun, L., Fan, Z., Wang, Y., Huang, Y., Schmidt, M. and Zhang, M. (2015) Tunable Synthesis of Self-Assembled Cyclic Peptide Nanotubes and Nanoparticles. Soft Matter, 11, 3822-3832. https://doi.org/10.1039/C5SM00533G

[19] Wang, Y., Yi, S., Sun, L., Huang, Y. and Zhang, M. (2014) Charge-Selective Fractions of Naturally Occurring Nanoparticles as Bioactive Nanocarriers for Cancer Therapy. Acta Biomaterialia, 10, 4269-4284.

https://doi.org/10.1016/j.actbio.2014.06.020

[20] Sun, L., Huang, Y., Bian, Z., Petrosino, J., Fan, Z. and Fan, Y., et al. (2016) Sundew-Inspired Adhesive Hydrogels Combined with Adipose-Derived Stem Cells for Wound Healing. ACS Applied Materials \& Interfaces, 8, 2423-2434.

https://doi.org/10.1021/acsami.5b11811

[21] Bratovcic, A. (2020) Biosynthesis of Green Silver Nanoparticles and Its UV-Vis Characterization. International Journal of Innovative Science, Engineering \& Technology, 7, 170-176.

[22] Abu-Dalo, M., Jaradat, A., Albiss, B.A. and Al-Rawashdeh, N.A.F. (2019) Green Synthesis of $\mathrm{TiO}_{2}$ NPs/Pristine Pomegranate Peel Extract Nanocomposite and Its Antimicrobial Activity for Water Disinfection. Journal of Environmental Chemical Engineering, 7, Article ID: 103370. https://doi.org/10.1016/j.jece.2019.103370

[23] Rai, M. and Kon, K. (2015) Nanotechnology in Diagnosis, Treatment and Prophylaxis of Infectious Diseases. Academic Press, Cambridge, $344 \mathrm{p}$.

[24] Bratovcic, A. (2019) Different Applications of Nanomaterials and Their Impact on the Environment. International Journal of Material Science and Engineering, 5, 1-7. https://doi.org/10.14445/23948884/IJMSE-V5I1P101

[25] Mihai, A.D., Chircov, C., Grumezescu, A.M. and Holban, A.M. (2020) Magnetite Nanoparticles and Essential Oils Systems for Advanced Antibacterial Therapies. International Journal of Molecular Sciences, 21, 7355. https://doi.org/10.3390/ijms21197355

[26] Bratovcic, A. (2020) Nanomaterials in Food Processing and Packaging, Its Toxicity and Food Labeling. Acta Scientific Nutritional Health, 4, 7-13.

[27] Mirkin, C.A., Letsinger, R.L., Mucic, R.C. and Storhoff, J.J. (1996) A DNA-Based Method for Rationally Assembling Nanoparticles into Macroscopic Materials. Nature, 382, 607-609. https://doi.org/10.1038/382607a0

[28] Silva, S.M., Tavallaie, R., Sandiford, L., Tilley, R.D. and Gooding, J.J. (2016) Gold Coated Magnetic Nanoparticles: From Preparation to Surface Modification for Analytical and Biomedical Applications. Chemical Communications, 52, 7528-7540. https://doi.org/10.1039/C6CC03225G

[29] Wu, W., Jiang, C.Z. and Roy, V.A.L. (2016) Designed Synthesis and Surface Engineering Strategies of Magnetic Iron Oxide Nanoparticles for Biomedical Applications. Nanoscale, 8, 19421-19474. https://doi.org/10.1039/C6NR07542H

[30] Kolosnjaj-Tabi, J., Lartigue, L., Javed, Y., Luciani, N., Pellegrino, T., Wilhelm, C., Alloyeau, D. and Gazeau, F. (2016) Biotransformations of Magnetic Nanoparticles in the Body. Nanotoday, 11, 280-284. https://doi.org/10.1016/j.nantod.2015.10.001

[31] Duguet, E., Vasseur, S., Mornet, S. and Devoisselle, J.M. (2006) Magnetic Nanopar- 
ticles and Their Applications in Medicine. Nanomedicine, 1, 157-168. https://doi.org/10.2217/17435889.1.2.157

[32] Gu, H.W., Xu, K.M., Xu, C.J. and Xu, B. (2006) Biofunctional Magnetic Nanoparticles for Protein Separation and Pathogen Detection. Chemical Communications, No. 9, 941-949. https://doi.org/10.1039/B514130C

[33] Yuen, C. and Liu, Q. (2012) Magnetic Field Enriched Surface Enhanced Resonance Raman Spectroscopy for Early Malaria Diagnosis. Journal of Biomedical Optics, 17, Article ID: 017005. https://doi.org/10.1117/1.JBO.17.1.017005

[34] Jeon, W., Lee S., Manjunatha, D. and Ban, C. (2013) A Colorimetric Aptasensor for the Diagnosis of Malaria Based on Cationic Polymers and Gold Nanoparticles. Analytical Biochemistry, 439, 11-16. https://doi.org/10.1016/j.ab.2013.03.032

[35] Zheng, T. and Huo, Q. (2020) A Nanoparticle Pseudo Pathogen for Rapid Detection and Diagnosis of Virus Infection. Sensors International, 1, Article ID: 100010. https://doi.org/10.1016/j.sintl.2020.100010

[36] Krampa, F.D., Aniweh, Y., Kanyong, P. and Awandare, G.A. (2020) Recent Advances in the Development of Biosensors for Malaria Diagnosis. Sensors International, 20, 799. https://doi.org/10.3390/s20030799

[37] Yetisgin, A.A., Cetinel, S., Zuvin, M., Kosar, A. and Kutlu, O. (2020) Therapeutic Nanoparticles and Their Targeted Delivery Applications. Molecules, 25, 2193. https://doi.org/10.3390/molecules25092193

[38] Zazo, H., Colino, C.I. and Lanao, J.M. (2016) Current Applications of Nanoparticles in Infectious Diseases. Journal of Controlled Release, 224, 86-102. https://doi.org/10.1016/j.jconrel.2016.01.008

[39] Volpedo, G., Costa, L., Ryan, N., Halsey, G., Satoskar, A. and Oghumu, S. (2019). Nanoparticulate Drug Delivery Systems for the Treatment of Neglected Tropical Protozoan Diseases. Journal of Venomous Animals and Toxins including Tropical Diseases, 25, e144118. https://doi.org/10.1590/1678-9199-jvatitd-1441-18

[40] Mehrizi, T.Z., Ardestani, M.S., Hoseini, M.H.M., Khamesipour, A., Mosaffa, N. and Ramezani, A. (2018) Novel Nanosized Chitosan-Betulinic Acid Against Resistant Leishmania Major and First Clinical Observation of Such Parasite in Kidney. Scintific Reports, 8, Article No. 11759. https://doi.org/10.1038/s41598-018-30103-7

[41] Ahmadpour, E., Godrati-Azar, Z., Spotin, A., Norouzi, R., Hamishehkar, H., Nami, S., Heydarian, P., Rajabi, S., Mohammadi, M. and Perez-Cordon, G. (2019) Nanostructured Lipid Carriers of Ivermectin as a Novel Drug Delivery System in Hydatidosis. Parasites \& Vectors, 12, Article No. 469.

https://doi.org/10.1186/s13071-019-3719-x

[42] Kowouvi, K., Alies, B., Gendrot, M., Gaubert, A., Vacher, G. and Gaudin, K., et al. (2019) Nucleoside-Lipid-Based Nanocarriers for Methylene Blue Delivery: Potential Application as Anti-Malarial Drug. RSC Advances, 9, 18844-18852. https://doi.org/10.1039/C9RA02576F

[43] Yazdian-Robati, R., Hedayati, N., Ramezani, M., Abnous, K. and Taghdisi, S.M. (2018) Colorimetric Gold Nanoparticles-Based Aptasensors. Nanomedicine Journal, $5,1-5$.

[44] Borgheti-Cardoso, L.N., Anselmo, M.S., Lantero, E., Lancelot, A., Serrano, J.L., Hernández-Ainsa, S., Fernàndez-Busquets, X. and Sierra, T. (2020) Promising Nanomaterials in the Fight against Malaria. Journal of Materials Chemistry B, 8 , 9428-9448. https://doi.org/10.1039/D0TB01398F

[45] Vassallo, A., Silletti, M.F., Faraone, I. and Milella, L. (2020) Nanoparticulate Antibiotic Systems as Antibacterial Agents and Antibiotic Delivery Platforms to Fight 
Infections. Journal of Nanomaterials, 2020, Article ID: 6905631. https://doi.org/10.1155/2020/6905631

[46] Coma-Cros, E.M., Biosca, A., Lantero, E., Manca, M.L., Caddeo, C., Gutiérrez, L., Ramírez, M., Borgheti-Cardoso, L.N., Manconi, M. and Fernàndez-Busquets, X. (2018) Antimalarial Activity of Orally Administered Curcumin Incorporated in Eudragit ${ }^{\circledR}$-Containing Liposomes. International Journal of Molecular Sciences, 19, 1361. https://doi.org/10.3390/ijms19051361

[47] Weynom, E.J., Badawi, M.M., Yisa, A., Gana, B., Okorie, M., Itodo, G. and Adeniyi, K.A. (2019) Nanotechnology for Improved Anti-Malaria Efficacy: Review Update. International Journal of Cell Science \& Molecular Biology, 6, 555690.

[48] Varela-Aramburu, S., Ghosh, C., Goerdeler, F., Priegue, P., Moscovitz, O. and Seeberger, P.H. (2020) Targeting and Inhibiting Plasmodium falciparum Using Ultra-Small Gold Nanoparticles. ACS Applied Materials \& Interfaces, 12, 43380-43387. https://doi.org/10.1021/acsami.0c09075

[49] Dkhil, M.A., Khalil, M.F., Diab, M.S.M., Bauomy, A.A. and Al-Quraishy, S. (2017) Effect of Gold Nanoparticleson Mice Splenomegaly Induced by Schistosomiasis Mansoni. Saudi Journal of Biological Sciences, 24, 1418-1423.

https://doi.org/10.1016/j.sjbs.2016.12.017

[50] Dkhil, M.A., Khalil, M.F., Diab, M.S.M., Bauomy, A.A., Santourlidis, S., Al-Shaebi, E.M. and Al-Quraishy, S. (2019) Evaluation of Nanoselenium and Nanogold Activities against Murine Intestinal Schistosomiasis. Saudi Journal of Biological Sciences, 26, 1468-1472. https://doi.org/10.1016/j.sjbs.2018.02.008

[51] Kar, P.K., Murmu, S., Saha, S., Tandon, V. and Acharya, K. (2014) Anthelmintic Efficacy of Gold Nanoparticles Derived from a Phytopathogenic Fungus, Nigrospora oryzae. PLoS ONE, 9, e84693. https://doi.org/10.1371/journal.pone.0084693

[52] Saad, H., Soliman, M.I., Azzam, A.M. and Mostafa, B. (2015) Antiparasitic Activity of Silver and Copper Oxide Nanoparticles against Entamoeba histolytica and Cryptosporidium parvum Cysts. Journal of the Egyptian Society of Parasitology, 45, 593-602. https://doi.org/10.12816/0017920

[53] Roy, P., Saha, S.K., Gayen, P., Chowdhury, P. and Babu, S.P.S. (2018) Exploration of Antifilarial Activity of Gold Nanoparticle against Human and Bovine Filarial Parasites: A Nanomedicinal Mechanistic Approach. Colloids and Surfaces B: Biointerfaces, 161, 236-243. https://doi.org/10.1016/j.colsurfb.2017.10.057

[54] Hikal, W.M., Said-Al Ahl, H.A.H. and Tkachenko, K.G. (2020) Present and Future Potential of Antiparasitic Activity of Opuntia ficus-indica. Tropical Journal of Natural Product Research, 4, 672-679. https://doi.org/10.26538/tjnpr/v4i10.3

[55] Adeyemi, O.S., Molefe, N.I., Awakan, O.J., Nwonuma, C.O., Alejolowo, O.O., Olaolu, T., Maimako, R.F., Suganuma, K., Han, Y. and Kato, K. (2018) Metal Nanoparticles Restrict the Growth of Protozoan Parasites. Artificial Cells, Nanomedicine, and Biotechnology, 46, S86-S94.

https://doi.org/10.1080/21691401.2018.1489267

[56] Nafari, A., Cheraghipour, K., Sepahvand, M., Shahrokhi, G., Gabal, E. and Mahmoudvand, H. (2020) Nanoparticles: New Agents toward Treatment of Leishmaniasis. Parasite Epidemiology and Control, 10, e00156. https://doi.org/10.1016/j.parepi.2020.e00156

[57] Vazini, H. (2018) The In Vitro and In Vivo Efficacy of Gold Nanoparticle in Comparison to the Glucantime as a Therapeutic Agent against L. Major. Journal of Infectious Diseases and Therapy, 6, 373. https://doi.org/10.4172/2332-0877.1000373

[58] Morilla, M.J. and Romero, E.L. (2015) Nanomedicines against Chagas Disease: An 
Update on Therapeutics, Prophylaxis and Diagnosis. Nanomedicine, 10, 465-481. https://doi.org/10.2217/nnm.14.185

[59] Baranwal, A., Chiranjivi, A.K., Kumar, A., Dubey, V.K. and Chandra, P. (2018) Design of Commercially Comparable Nanotherapeutic Agent against Human Disease-Causing Parasite, Leishmania. Scientific Reports, 8, Article No. 8814. https://doi.org/10.1038/s41598-018-27170-1

[60] Kumar, P., Shivam, P., Mandal, S., Prasanna, P., Kumar, S., Prasad, S.R., Kumar, A., Das, P., Ali, V., Singh, S.K. and Mandal, D. (2019) Synthesis, Characterization, and Mechanistic Studies of a Gold Nanoparticle-Amphotericin B Covalent Conjugate with Enhanced Antileishmanial Efficacy and Reduced Cytotoxicity. International Journal of Nanomedicine, 14, 6073-6101. https://doi.org/10.2147/IJN.S196421

[61] Fox, S. (2019) Gold Nanoparticles and Lasers Kill the Brain Parasite That Causes "Crazy Cat Lady" Syndrome. https://www.popsci.com/

https://www.popsci.com/science/article/2010-03/gold-nanoparticles-and-lasers-killcrazy-cat-lady-brain-parasite/

[62] Abdulsattar, S.A. and Drwall, A.H. (2017) Effect of Gold and Nickel Nanoparticles on Immune Proteins of Toxoplasmosis Patients. Asian Journal of Pharmaceutical and Clinical Research, 10, 162-164.

[63] Said, D., Elsamad, L. and Gohar, Y. (2012) Validity of Silver, Chitosan, and Curcumin Nanoparticles as Anti-Giardia Agents. Parasitology Research, 111, 545-554. https://doi.org/10.1007/s00436-012-2866-1

[64] Bavand, Z., Gholami, S., Honary, S., Rahimi, E.B., Torabi, N. and Barabadi, H. (2014) Effect of Gold Nanoparticles on Giardia Lamblia Cyst Stage in In Vitro. Journal of Arak University of Medical Sciences, 16, 27-37.

[65] Sedighi, F., Abbasali, P.R., Maghsood, A. and Fallah, M. (2016) Comparison of Therapeutic Effect of Anti-Cryptosporidium Nano-Nitazoxanide (ntz) with Free Form of This Drug in Neonatal Rat. Scientific Journal of Hamadan University of Medical Sciences, 23, 134-140.

[66] Allahverdiyev, A.M., Abamor, E.S., Bagirova, M., Ustundag, C.B., Kaya, C., Kaya, F., et al. (2011) Antileishmanial Effect of Silver Nanoparticles and Their Enhanced Antiparasitic Activity under Ultraviolet Light. International Journal of Nanomedicine, 6, 2705. https://doi.org/10.2147/IJN.S23883

[67] Allahverdiyev, A.M., Abamor, E.S., Bagirova, M. and Rafailovich, M. (2011) Antimicrobial Effects of $\mathrm{TiO}_{2}$ and $\mathrm{Ag}_{2} \mathrm{O}$ Nanoparticles against Drug-Resistant Bacteria and Leishmania Parasites. Future Microbiology, 6, 933-940.

https://doi.org/10.2217/fmb.11.78

[68] Sazgarnia, A., Taheri, A.R., Soudmand, S., Parizi, A.J., Rajabi, O. and Darbandi, M.S. (2013) Antiparasitic Effects of Gold Nanoparticles with Microwave Radiation on Promastigotes and Amastigotes of Leishmania major. International Journal of Hyperthermia, 29, 79-86. https://doi.org/10.3109/02656736.2012.758875

[69] Venier-Julienne, M., Vouldoukis, I., Monjour, L. and Benoit, J. (1995) In Vitro Study of the Anti-Leishmanial Activity of Biodegradable Nanoparticles. Journal of Drug Targeting, 3, 23-29. https://doi.org/10.3109/10611869509015929

[70] Salah-Tazdaï, R, Tazdaït, D, Harrat, Z, Eddaikra, N, Abdi, N. and Mameri, N. (2015) Antiparasite Activity of Chitosan. Proceedings of 2015 International Conference on Chemical, Metallurgy and Environmental Engineering, Istanbul, 3-4 June 2015, 277-280.

[71] Karimi, M., Dalimi, A., Jamei, F., Ghaffarifar, F. and Dalimi, A. (2015) The Killing Effect of Silver Nanoparticles and Direct Electric Current Induction on Leishmania 
major Promastigotes in Vitro. Modares Journal of Medical Sciences. Pathobiology, 18, 87-96.

[72] Khosravi, A., Sharifi, I., Barati, M., Zarean, M. and Hakimi-Parizi, M. (2011) Anti-Leishmanial Effect of Nanosilver Solutions on Leishmania tropica Promastigotes by in-Vitro Assay. Zahedan Journal of Research in Medical Sciences, 13, 8-12.

[73] Jameii, F., Dalimi Asl, A., Karimi, M. and Ghaffarifar, F. (2015) Healing Effect Comparison of Selenium and Silver Nanoparticles on Skin Leishmanial Lesions in Mice. Scientific Journal of Hamadan University of Medical Sciences, 22, 217-223.

[74] Gaafar, M., Mady, R., Diab, R. and Shalaby, T.I. (2014) Chitosan and Silver Nanoparticles: Promising Anti-Toxoplasma Agents. Experimental Parasitology, 143, 30-38. https://doi.org/10.1016/j.exppara.2014.05.005

[75] Mohapatra, S.C., Tiwari, H.K., Singla, M., Rathi, B., Sharma, A., Mahiya, K., et al. (2010) Antimalarial Evaluation of Copper (II) Nanohybrid Solids: Inhibition of Plasmepsin II, a Hemoglobin-Degrading Malarial Aspartic Protease from Plasmodium falciparum. JBIC Journal of Biological Inorganic Chemistry, 15, 373-385. https://doi.org/10.1007/s00775-009-0610-9

[76] Tripathy, S., Das S., Chakraborty, S.P., Sahu, S.K., Pramanik, P. and Roy, S. (2012) Synthesis, Characterization of chitosan-Tripolyphosphate Conjugated Chloroquine Nanoparticle and Its In Vivo Anti-Malarial Efficacy against Rodent Parasite: A Dose and Duration Dependent Approach. International Journal of Pharmaceutics, 434, 292-305. https://doi.org/10.1016/j.ijpharm.2012.05.064

[77] Ponarulselvam, S., Panneerselvam, C., Murugan, K., Aarthi, N., Kalimuthu, K. and Thangamani, S. (2012) Synthesis of Silver Nanoparticles Using Leaves of Catharanthus roseus Linn. G. Don and their Antiplasmodial Activities. Asian Pacific Journal of Tropical Biomedicine, 2, 574-580.

https://doi.org/10.1016/S2221-1691(12)60100-2

[78] Nayak, A.P., Tiyaboonchai, W., Patankar, S., Madhusudhan, B. and Souto, E.B. (2010) Curcuminoids-Loaded Lipid Nanoparticles: Novel Approach towards Malaria Treatment. Colloids and Surfaces B: Biointerfaces, 81, 263-273. https://doi.org/10.1016/j.colsurfb.2010.07.020

[79] Abulaihaiti, M., Wu, X.W., Qiao, L., Lv, H.L., Zhang, H.W., Aduwayi, N., et al. (2015). Efficacy of Albendazole-Chitosan Microsphere-Based Treatment for Alveolar Echinococcosis in Mice. PLoS Neglected Tropical Diseases, 9, e0003950. https://doi.org/10.1371/journal.pntd.0003950

[80] Brodaczewska, K., Wolaniuk, N., Donskow-Lysoniewska, K. and Doligalska, M. (2013) Chitosan Stimulates Lymphocyte Proliferation during the Muscle Phase of Trichinella spiralis Infection in Mice. 15th International Congress of Immunology, Milan, 22-27 August 2013. https://doi.org/10.3389/conf.fimmu.2013.02.01117

[81] Gherbawy, Y.A., Shalaby, I.M., El-sadek, M.S.A., Elhariry, H.M. and Banaja, A.A. (2013) The Anti-Fasciolasis Properties of Silver Nanoparticles Produced by Trichoderma harzianum and Their Improvement of the Anti-Fasciolasis Drug Triclabendazole. International Journal of Molecular Sciences, 14, 21887-21898.

https://doi.org/10.3390/ijms141121887 